\title{
EL PRÁCTICUM EN EDUCACIÓN FÍSICA EN EL MEDIO NATURAL: CONECTANDO ESPACIOS FÍSICOS Y VIRTUALES
}

\author{
O PRACTICUM NA EDUCAÇÃO FÍSICA NO AMBIENTE NATURAL: \\ CONECTANDO ESPAÇOS FÍSICOS E VIRTUAIS
}

PHYSICAL EDUCATION PRACTICUM IN THE NATURAL ENVIRONMENT: LINKING PHYSICAL AND VIRTUAL SPACES

\author{
Vanesa Gallego-Lema*, Juan Alberto Muñoz-Cristóbal**, \\ Higinio Francisco Arribas-Cubero**, Bartolomé Rubia-Avi**
}

\section{Palabras clave: \\ Tecnología \\ educacional. \\ Actividad motora. \\ Educación Primaria \\ y Secundaria. \\ Educación Superior.}

\begin{abstract}
Resumen: Los maestros en formación que acuden al Prácticum vinculan los conocimientos adquiridos en la Universidad para llevarlos a la práctica en las escuelas, siendo relevante esta etapa para conocer la aplicación que hacen de la competencia digital en el Prácticum de Educación Primaria. La investigación se centró en analizar, mediante un estudio de caso, cómo estudiantes universitarios aplican la formación recibida en tecnologías ubicuas en la asignatura Educación Física en el Medio Natural a la escuela durante su Prácticum. Entre otros resultados, las evidencias mostraron aspectos que hicieron que la integración tecnológica durante el Prácticum fuera distinta entre los estudiantes (p.ej. background tecnológico del centro). Al incluir las TIC emergieron potencialidades (p.ej. la ubicuidad del aprendizaje) y dificultades (p.ej. problemas tecnológicos), las cuales iluminaron los aspectos a tener en cuenta en la aplicabilidad de los aprendizajes tecnológicos en el campo de las actividades físicas en el medio natural.
\end{abstract}

\section{Palavras chave}

Tecnologia

educacional.

Atividade motora.

Ensino fundamental

e médio.

Educação Superior.
Resumo: Os professores em formação que frequentam o Practicum vinculam os conhecimentos adquiridos na Universidade para colocá-los em prática nas escolas, sendo este estágio relevante para conhecer a aplicação que eles fazem da competência digital no Practicum realizado na Educação Primária. A pesquisa centrou-se na análise, através de um estudo de caso, de como os estudantes universitários aplicam o treinamento recebido em tecnologias ubíquas na disciplina de Educação Física no Meio Ambiente Natural na escola durante suas práticas. Entre outros resultados, as evidências mostraram aspectos que tornaram a integração tecnológica durante o Practicum diferente entre os alunos (p.ex., o background tecnológico do centro). Ao incluir as TIC, surgiram potencialidades (p.ex., a ubiquidade da aprendizagem) e as dificuldades (p.ex., problemas tecnológicos) que iluminaram os aspectos a serem considerados na aplicabilidade da aprendizagem tecnológica no campo das atividades físicas no ambiente natural.

Abstract: Student teachers doing Practicum connect knowledge acquired at the University and apply it in schools. The activity is important for knowing their use of digital competence during Practicum in Elementary Education. This study focused on analyzing, my means of a case study, how student teachers apply their training in ubiquitous technologies they received during the course Physical Education in the Natural Environment to their practicum in the schools. Among other findings, the evidence showed aspects which made technological integration distinct between students during Practicum (e.g. the school's technological background). By including ICTs, there emerged potentialities (e.g., ubiquity of learning) and difficulties (e.g. technological problems) that shed light on the aspects to be considered in the applicability of technological learning to the field of physical activities in the natural environment.
*Universidad del País Vasco. San Sebastián. España.

E-mail: vanesa.gallego@ehu.eus

**Universidad de Valladolid. Valladolid. España.

E-mail: juanmunoz@gsic.uva.es; quico@mpc.uva.es; brubia@pdg.uva.es

Recebido em: 14-04-2018 Aprovado em: 06-03-2018 Publicado em: 18-04-2019

DOI: https://doi.org/10.22456/1982-8918.81980 (c) (1) \&) Licence 


\section{INTRODUCCION}

La formación de los futuros docentes en el periodo de prácticas que realizan en los centros escolares (denominado Prácticum en los programas universitarios españoles), tiene un papel muy importante en su aprendizaje ya que durante esta etapa los estudiantes están en relación directa con la realidad educativa, después de un proceso de aprendizaje teóricopráctico en la universidad (ZABALZA, 2011). Para Bunk (1994) este proceso requiere una formación que se relacione con las tareas profesionales, con la finalidad de que transmitir competencias tenga un sentido global. Por otro lado, incidir también en la relevancia del periodo de prácticas debido a que los futuros docentes (re)construyen su identidad profesional, gracias a un trabajo crítico y reflexivo mediante la observación y el análisis de sus prácticas (GONZÁLEZ; BARBA; RODRíGUEZ, 2015). No obstante, es necesario reflexionar cómo los aprendizajes que reciben los estudiantes en la universidad son aplicables cuando acuden a la escuela. En este sentido, Sancho-Gil y Correa-Gorospe (2015) advierten de la necesidad de revisar la relación entre la formación y la práctica docente en los procesos formativos para que los futuros maestros aprendan a ser docentes; mientras que Tejada y Ruiz (2013) inciden en que el docente universitario no debe ceñirse únicamente a la transmisión de conocimientos, leyes, etc., sino pasar del saber a la acción para que los estudiantes adquieran una competencia profesional.

Uno de los grandes desafíos que se está produciendo en la Educación Superior es la incorporación de las Tecnologías de la Información y la Comunicación (TIC) (ZABALZA, 2011), aunque existen ya experiencias en la formación inicial del profesorado satisfactorias en este sentido (PRAT; CAMERINO; COIDURAS, 2013). La inclusión de las TIC y la competencia digital en las universidades es una línea prioritaria de acuerdo con el documento marco para la convergencia de la universidad española en el Espacio Europeo (MINISTERIO DE EDUCACIÓN, 2003; MARGALEF; ÁLVAREZ, 2005), así como una de las competencias integradas en el currículum de Educación Primaria actual (Ley Orgánica para la Mejora de la Calidad Educativa - LOMCE). Sin embargo, la práctica diaria está bastante alejada de los ideales educativos así como de la normativa (GUTIÉRREZ; PALACIOS; TORREGO, 2010), encontrando dentro de este ámbito personas resistentes al uso tecnológico y a su vez, una escasa formación de los docentes. Como consecuencia de esto, se están llevando a cabo inversiones en los centros de formación, aunque en ocasiones estos recursos son poco utilizados (VÁZQUEZ-CANO; SEVILLANO, 2015). Otra de las limitaciones son las disonancias existentes entre el uso personal que los estudiantes universitarios realizan y sus creencias sobre el valor que tienen las TIC respecto a la aplicación que hacen de éstas en la docencia, ya que frecuentemente no se plasma en las programaciones didácticas en la escuela. Por ello, sería conveniente que en los planes de formación de profesorado universitario se abordará la competencia digital de manera transversal, desde distintas áreas, además de las específicas de TIC (PÉREZ; VÍLCHEZ, 2013), promoviendo un trabajo que conecten los ámbitos formal e informal, bajo el efecto de una cultura de colaboración, de las redes sociales, de accesibilidad a la información gracias a las tecnologías móviles.

En este sentido, el aprendizaje ubicuo ${ }^{1}$ puede dar respuesta a las necesidades de la actual sociedad contemporánea y abordar la competencia digital dentro de la formación

$1 \mathrm{El}$ aprendizaje ubicuo es aquel conocimiento que se da en cualquier lugar y momento a través de las TIC (COPE; KALANTZIS, 2010), y que supone trasladar el aula tradicional a otros contextos mediante el uso de herramientas tecnológicas en diferentes espacios y tiempos. 
inicial del profesorado. Actualmente, por medio de los dispositivos móviles, cualquier espacio físico puede conectarse a otro espacio virtual o real. El conocimiento y los espacios en los que se puede encontrar están evolucionando, de tal manera que cualquier persona pueda crear e irradiar información, así como aprender en cualquier momento y lugar gracias a los avances tecnológicos, llevándose a cabo un aprendizaje ubicuo (COPE; KALANTZIS, 2010). Esta evolución también altera la conexión tradicional existente entre docentes y alumnado, siendo ahora los estudiantes creadores y publicadores de contenido en redes sociales, blogs, etc., manteniendo una relación con el entorno más próximo (VÁZQUEZ-CANO; SEVILLANO, 2015) y convirtiéndose el docente en un colaborador del conocimiento situado del estudiante (JORRÍN-ABELLÁN; STAKE, 2009). Este proceso se ha convertido en un aprendizaje ubicuo, ya que a través de la integración de los móviles y de las redes sociales (BURBULES, 2012) se facilita la interacción y la información en infinidad de lugares y circunstancias.

Esta integración y aplicación de herramientas tecnológicas es peculiar en el área de Educación Física, ya que las actividades motrices se llevan a cabo en distintos espacios, siendo por tanto el uso de la tecnología diferente y complejo. Además, el profesorado de esta asignatura lleva a cabo actividades físicas en el medio natural bajo determinadas limitaciones, que pueden ser la raíz de que se utilicen menos las TIC en esta área que en otras, como son: la escasez de horas de la asignatura, miedo de pérdida de momentos motrices a favor de la tecnología, la necesidad de formación tecnológica y metodológica, la escasa cobertura en espacios naturales, la dificultad de llevar aparatos tecnológicos al estar en movimiento, etc. (PRAT et al., 2013; GALLEGO-LEMA et al., 2017). A pesar de ello, se están integrando herramientas dentro del campo de Educación Física en el Medio Natural (EFMN) en Educación Primaria y/o en la formación inicial de maestros en Educación Superior que apoyan contenidos específicos, como por ejemplo: conocimiento de contenidos de orientación a través de tecnologías ubicuas en Educación Primaria (MUÑOZ-CRISTÓBAL et al., 2015) y con maestros de Educación Física en formación (GALLEGO-LEMA et al., 2016; 2017); desarrollo de las habilidades de orientación mediante el uso de códigos QR y un blog en Educación Primaria (FERNÁNDEZ; HERRERA-VIDAL; NAVARRO, 2015); incremento de la motivación y de la interiorización de contenidos a través del uso de clickers en la formación inicial de profesorado (RIVERO et al, 2016); y la unión de los contenidos, materiales, recursos de la asignatura de EF dentro de una aplicación móvil (QUEROL, 2016). Dado que las experiencias y estudios que incluyen actividades tecnológicas en este campo son concretas, sumado a las limitaciones citadas del área y de la etapa de Educación Primaria para incluir tecnología, nos parece relevante estudiar por qué a pesar de la formación digital que se aporta a los futuros maestros en la Educación Superior, del habitual uso de las TIC en nuestra sociedad así como del apoyo legislativo y de los documentos marco, la aplicación de la competencia digital en los escuelas es tan diversa y parcial.

Así, en este trabajo pretendemos dar respuesta a la pregunta de investigación: ¿Cómo los estudiantes universitarios aplican la formación digital recibida durante la asignatura EFMN a la escuela durante su Prácticum? Para ello, realizamos un estudio de caso durante el Prácticum en EFMN, analizando la transferencia de la competencia digital en tres Centros de Educación Primaria en el curso 2013/2014. 


\section{DISEÑO DE LA INVESTIGACIÓN}

La investigación se centra en el periodo de Prácticum del Grado de Educación Primaria, con mención en Educación Física en una Universidad española. Previamente, los estudiantes cursaron la asignatura de EFMN, donde el docente implementó tecnologías ubicuas que facilitaron la realización de actividades más allá del aula ordinaria, como en el medio natural (parques urbanos, montaña, etc.) y otros espacios (casa, calle, etc.). Así, se incorporaron smartphones y tablets llevándose a cabo actividades con Realidad Aumentada, redes sociales y otras aplicaciones móviles que conectaron los distintos espacios físicos y virtuales (GALLEGO-LEMA et al., 2016; 2017). Una vez finalizada la asignatura de EFMN, un grupo de estudiantes aplicaron los aprendizajes adquiridos durante su Prácticum. Estos estudiantes son el foco del estudio, los cuales permitieron iluminar cómo se llevó a cabo la transferencia de la competencia digital adquirida durante su etapa universitaria al contexto escolar. La asignatura de EFMN fue impartida por un profesor con una experiencia docente de 22 años, con 65 estudiantes universitarios. Posteriormente, cuatro estudiantes de ese grupo realizaron el Prácticum y el Trabajo Fin de Grado (TFG) tutelados por el mismo profesor, muestra del estudio que presentamos.

Para ello, se analizó cómo los estudiantes aplicaron estos conocimientos en la etapa de Educación Primaria, siguiendo la perspectiva de estudio de caso de Stake (2005), que permitió explorar el problema de manera particular y contextualizada.

La Tabla 1 muestra las técnicas e instrumentos de recogida de datos que se utilizaron: observaciones, focus group y cuestionarios. De manera paralela, también obtuvimos datos por medio de documentos generados por los cuatro estudiantes universitarios, como fueron sus TFG, chats de carácter formativo de las sesiones; así como otros realizados por el alumnado de Educación Primaria mediante registros evaluativos en forma de puzles coloreables.

Tabla 1 - Detalle de las técnicas de recogida de datos utilizadas durante la investigación

\begin{tabular}{|c|c|}
\hline Técnica / acrónimo & Descripción \\
\hline Observación [Obs] & $\begin{array}{l}\text { Observaciones semi-estructuradas, realizadas por varios investigadores. Los datos } \\
\text { recogidos fueron audio/vídeo, fotografías, chats y notas, mediante las app Field Notes } \\
\text { Pro, Notes, Whatsapp, y la herramienta Learning Buckets. }\end{array}$ \\
\hline Focus [Focus] & $\begin{array}{l}\text { Conversaciones grupales cara a cara, semi-estructuradas, grabados mediante la app } \\
\text { Audio Memos en iPad. }\end{array}$ \\
\hline Cuestionario [Cuest] & $\begin{array}{l}\text { Cuestionarios compuestos de preguntas abiertas y cerradas, creados con Google } \\
\text { Drive. }\end{array}$ \\
\hline
\end{tabular}

Fuente: elaboración propia

Para recoger y sintetizar los datos se utilizaron las herramientas CSCL-EREM² (JORRÍNABELLÁN; STAKE, 2009) y Learning Buckets ${ }^{3}$ (MUÑOZ-CRISTÓBAL, 2015), facilitando la interacción entre los observadores y las observaciones, creando in situ los informes de las sesiones recogidas e incluyendo dentro contenido multimedia y textual geoposicionado. En la Figura 1 se puede observar el proceso de reducción y de asociación de los datos recogidos

2 Disponible en: http://pandora.tel.uva.es/cscl-erem/index.php. Acceso en: 29 mar 2019.

3 Disponible en: https://www.gsic.uva.es/buckets/ Acceso en: 29 mar 2019. 
que se siguió, buscando dar respuesta al issue (STAKE, 2005) o pregunta de investigación planteada. Con la finalidad de iluminar el issue, hemos propuesto un gran tema (declaración temática - DT): Formación y Prácticum. A su vez, dividimos la DT en diversas preguntas informativas $(\mathrm{PI})$ para profundizar en la declaración temática. Finalmente organizamos en categorías/temas los datos recogidos para dar respuesta a las PI, que son: competencia digital, aplicación digital en Primaria y TICs en la escuela.

Figura 1 - Estructura gráfica del proceso seguido de reducción y asociación de datos

(1): ¿Cómo los estudiantes universitarios aplican la formación digital recebida durante la
asignatura EFMN a la escuela durante su Prácticum?

Fuente: elaboración propia

La Figura 2 muestra la secuencia de investigación seguida. Durante los meses de abril y mayo (Acción 1-A1- en Figura 2) se impartió un curso de formación ad hoc a los cuatro estudiantes que el profesor de la asignatura Educación Física en el Medio Natural tuteló durante el Prácticum y Trabajo Fin de Grado (TFG) en el segundo cuatrimestre del curso 2013/2014. Los estudiantes universitarios ya utilizaron durante la asignatura EFMN las diferentes herramientas ubicuas, pero la realización de este proceso formativo aportó un mayor conocimiento de TIC de cara a la intervención didáctica durante su Prácticum. También se realizó un focus group con el alumnado para conocer su opinión sobre la utilización de estas herramientas durante la asignatura cursada en el primer cuatrimestre y cómo utilizarlas durante el Prácticum. Una vez que comenzaron el Prácticum en los tres Centros de Educación Primaria (A2), se recogieron datos de observación de más de 20 horas de actividades educativas. Además, investigadores y docente apoyaron durante estos meses, de manera virtual y presencial, las dudas de carácter tecnológico y didáctico que tenían los estudiantes. Por otro lado, tanto el profesor como el alumnado universitario respondieron a cuestionarios diferenciados, se analizaron sus TFG y los puzles evaluativos de los escolares para darles voz en el proceso de evaluación. 
Figura 2 - Secuencia de investigación seguida, indicando las principales acciones de investigación y las técnicas de recogida de datos empleadas

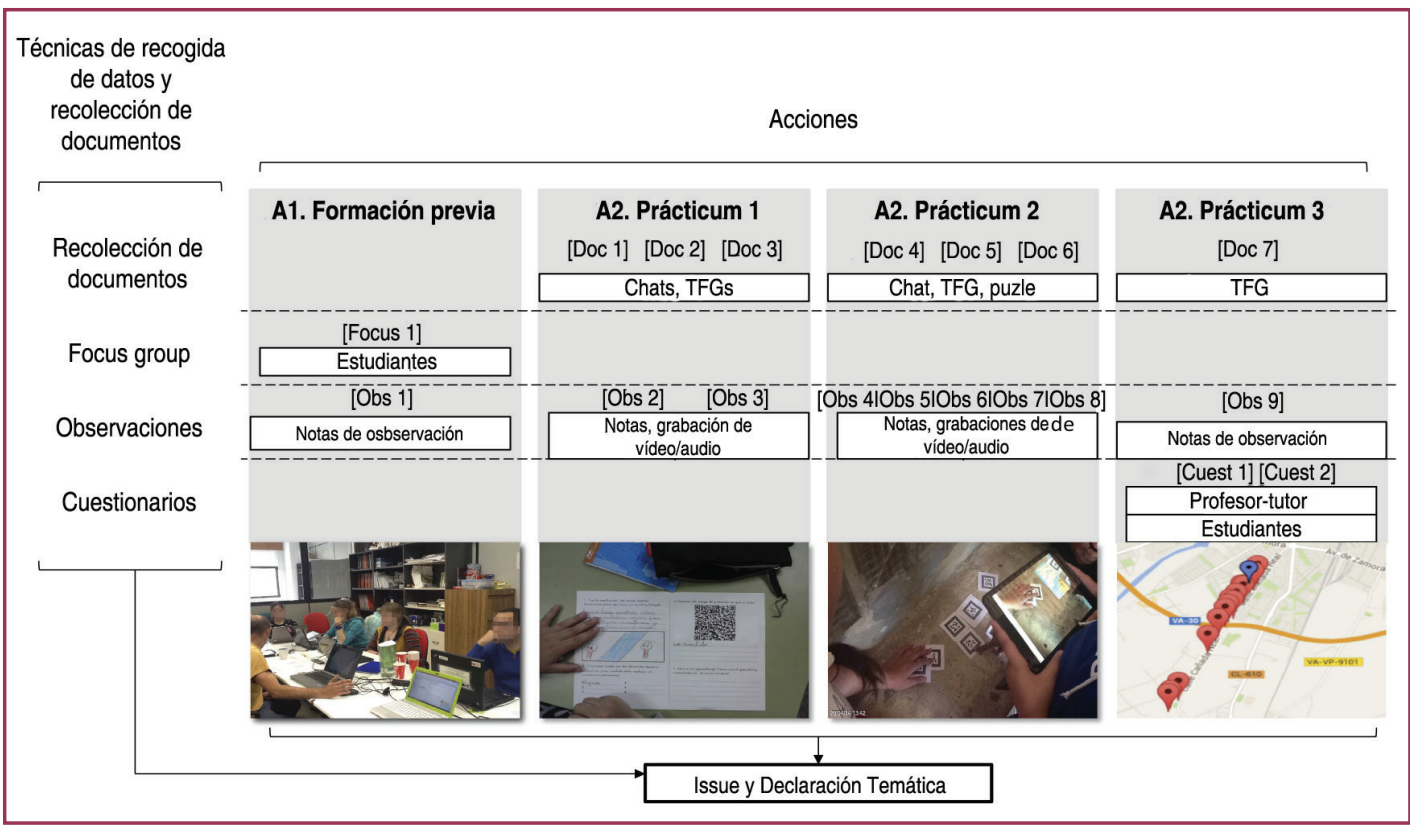

Fuente: elaboración propia

\section{INTERVENCIÓN DIDÁCTICA EN EL PRÁCTICUM}

En esta sección se describe la intervención realizada en los tres centros escolares de prácticas (Figuras 3, 4 y 5), donde cuatro estudiantes universitarios pusieron en práctica los conocimientos adquiridos durante el desarrollo de la asignatura EFMN y el curso ad hoc recibido:

Figura 3 - Intervención didáctica en el centro escolar 1

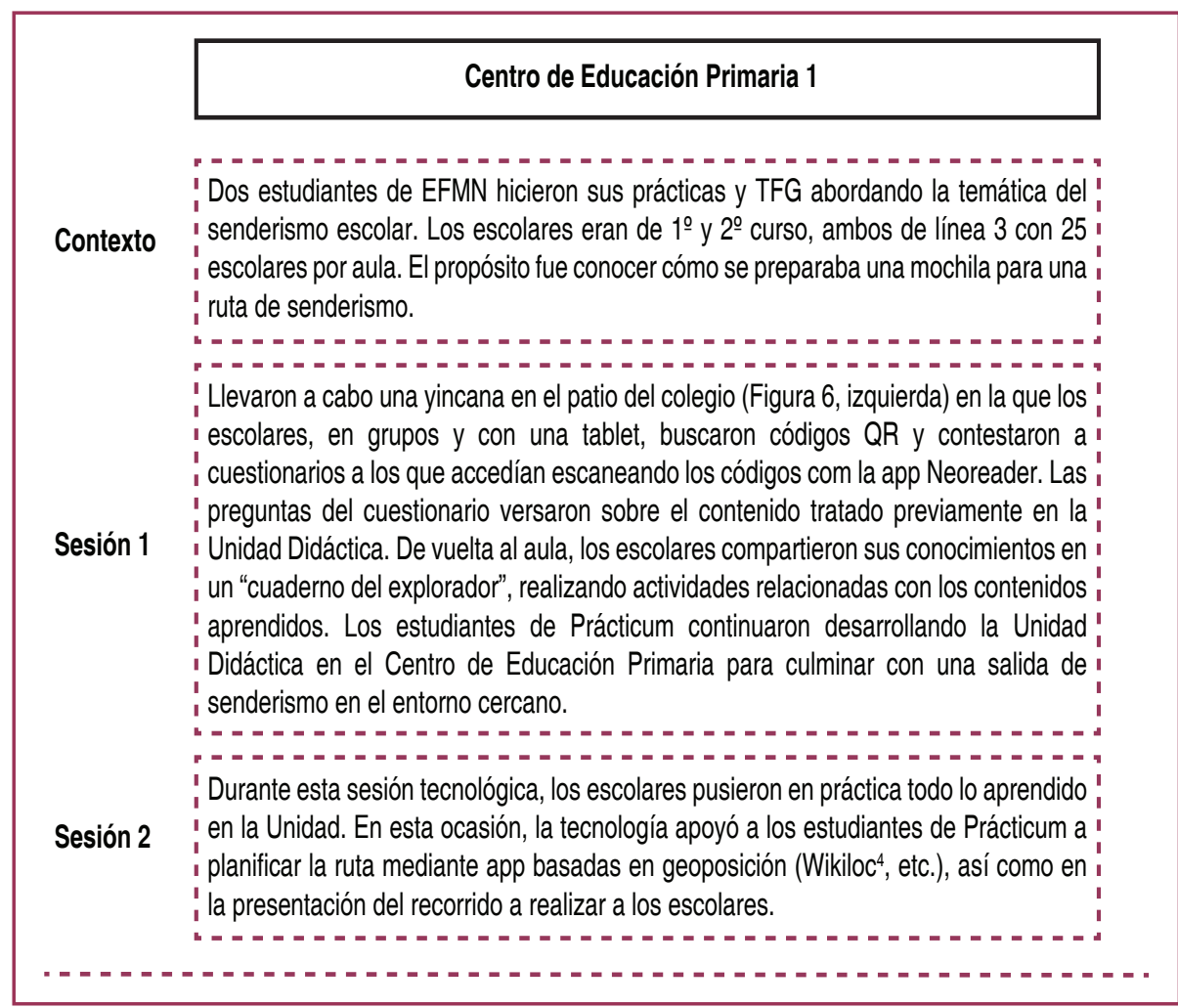

Fuente: elaboración propia

4 Disponible en: https://es. wikiloc.com/ Acceso en: 29 mar 2019. 
Figura 4 - Intervención didáctica en el centro escolar 2

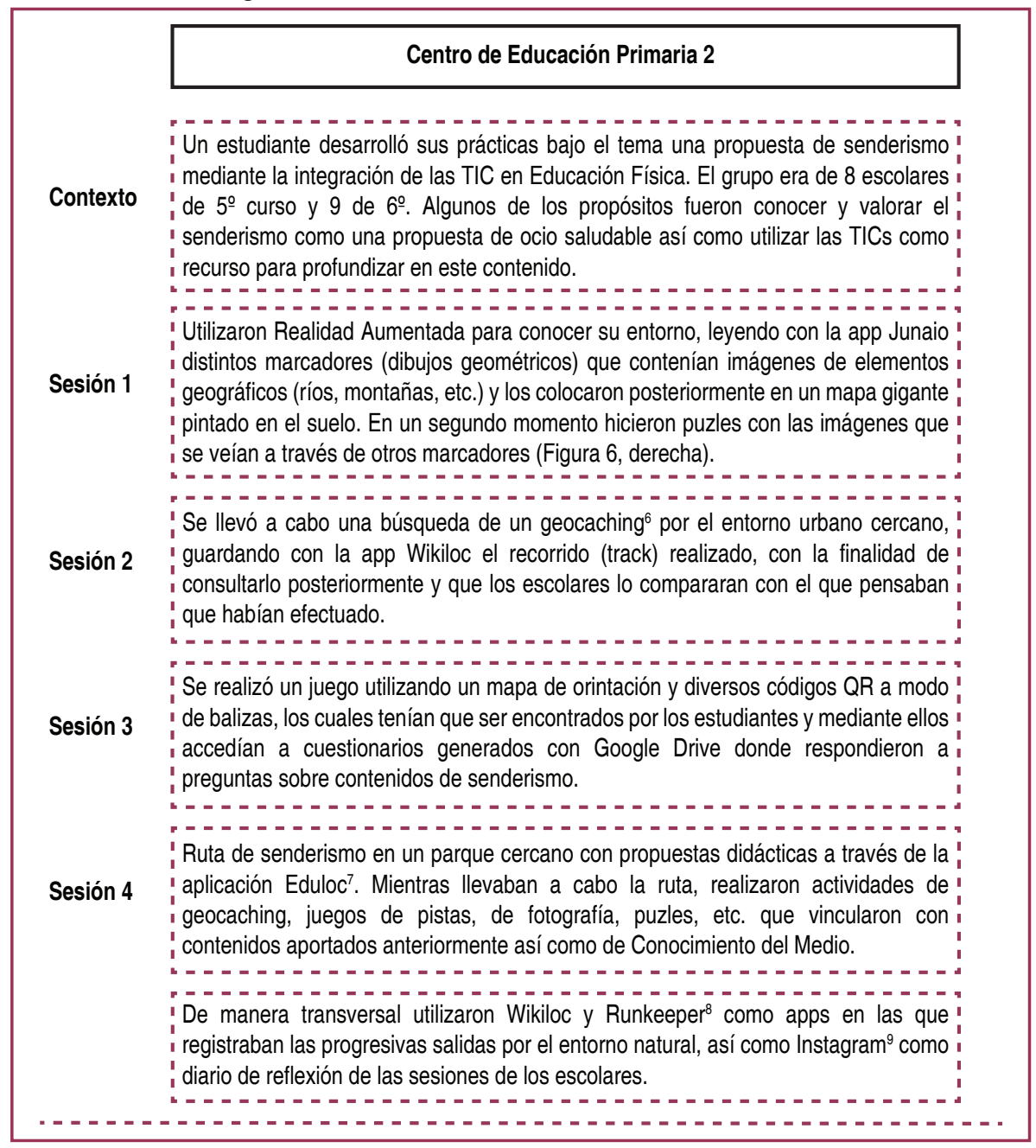

Fuente: elaboración propia

Figura 5 - Intervención didáctica en el centro escolar 3

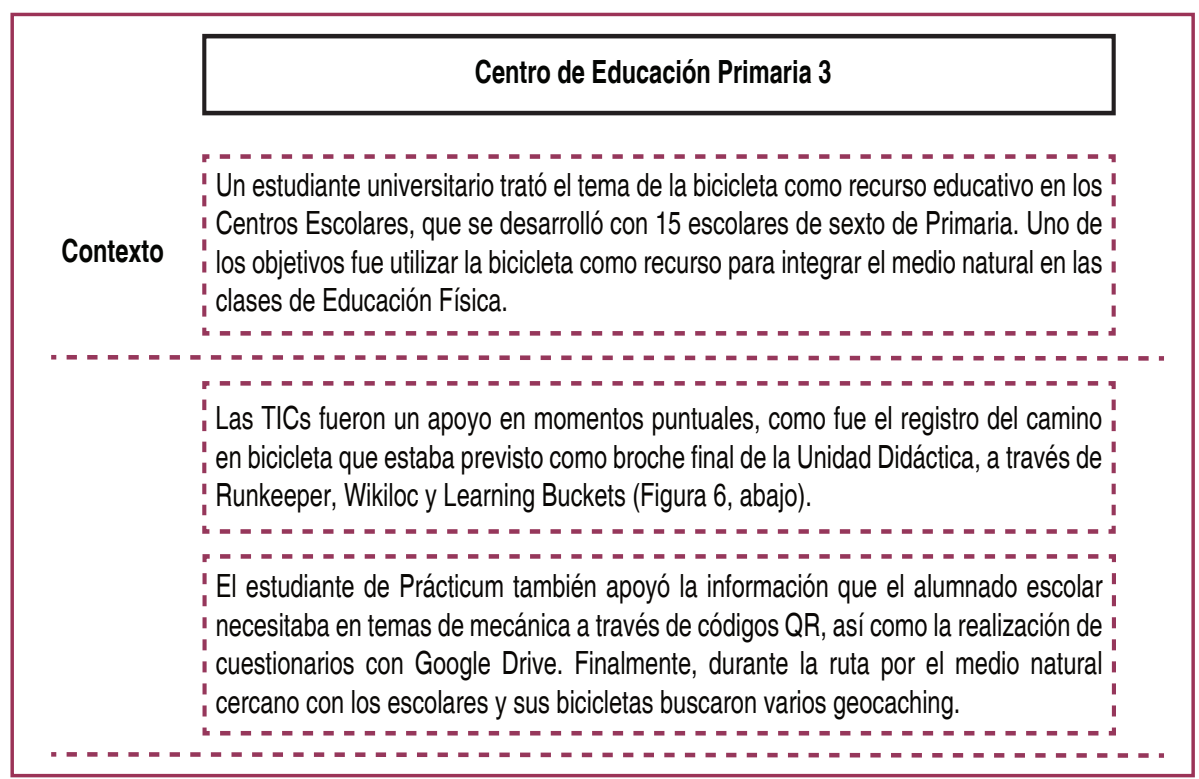

Fuente: elaboración propia

5 https://en.wikipedia.org/wiki/Junaio Acceso en: 29 mar 2019.

6 https://www.geocaching.com Acceso en: 29 mar 2019.

7 http://www.eduloc.net/es/escenari/2794/preview-iframe Acceso en: 29 mar 2019.

8 https://runkeeper.com/ Acceso en: 29 mar 2019.

9 https://www.instagram.com/ Acceso en: 29 mar 2019. 
Figura 6 - Desarrollo de la actividad en los Centros Escolares 1 (izquierda), 2 (derecha) y 3 (abajo)

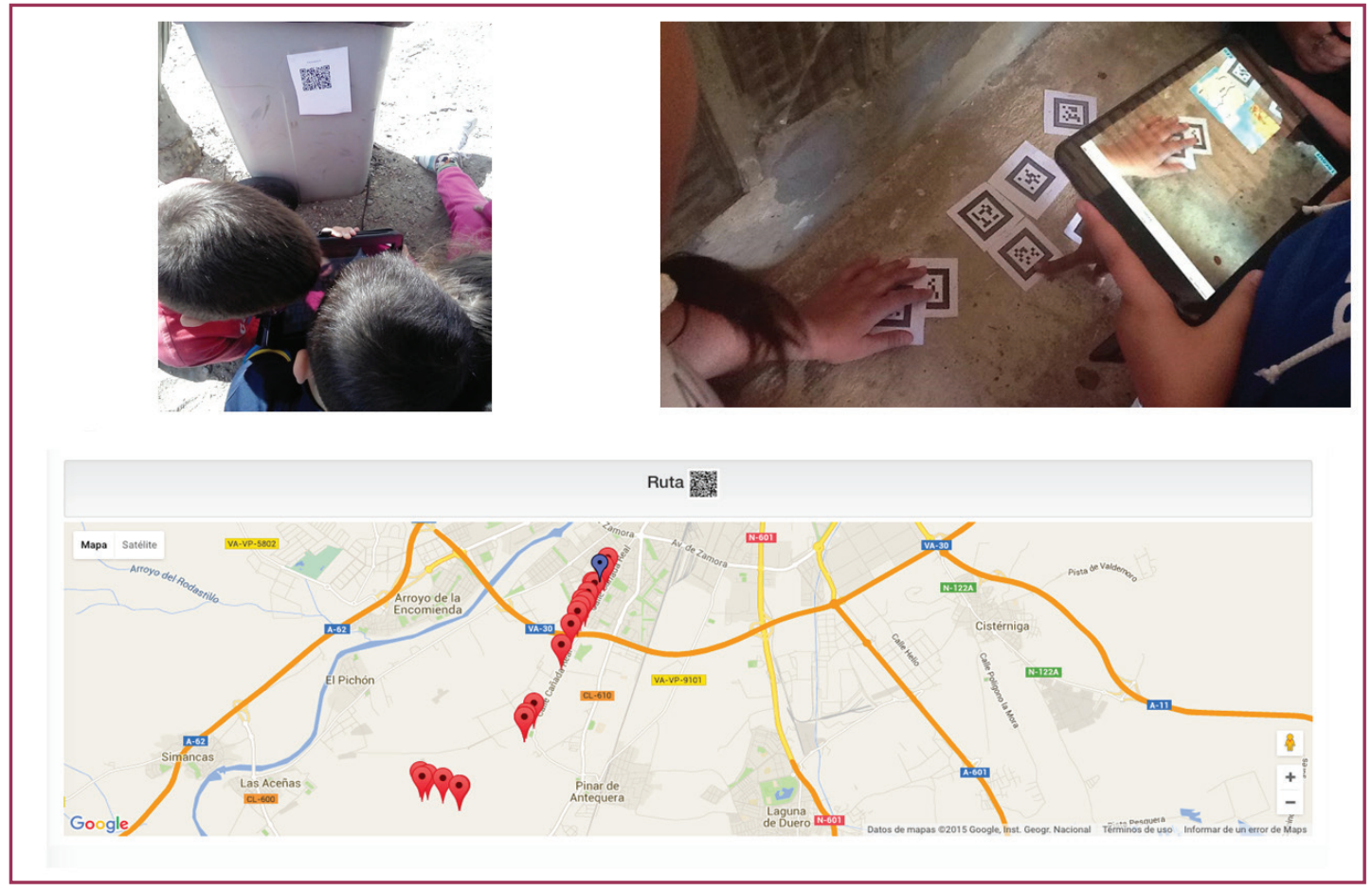

Fuente: elaboración propia

\section{RESULTADOS DE LA INVESTIGACIÓN}

En esta sección se describen los principales resultados obtenidos en el proceso de investigación, organizados según el esquema definido en el proceso de reducción de datos (Figura 1):

\subsection{COMPETENCIA DIGITAL}

En este apartado se analiza hasta qué punto la formación recibida por los estudiantes fue adecuada para que fueran competentes digitalmente y sus aprendizajes pudieran ser aplicados en Primaria. Así, el alumnado opinó que los contenidos tecnológicos tratados durante el curso fueron adecuados para llevarlos a cabo en el Prácticum, y que los aprendizajes que se desarrollaron en esta etapa de prácticas tuvieron relación con la realidad escolar después del proceso de aprendizaje en la universidad. La formación otorgada al alumnado sobre aprendizaje ubicuo y el uso de las diferentes herramientas tecnológicas que lo posibilitaron, les proporcionó autonomía a la hora de crear actividades con tecnología, sintiéndose preparados para realizar la intervención en los colegios. También hay que mencionar que fue un camino de adaptaciones en el cual el propio alumnado fue investigador, indagando en las posibilidades de las herramientas siempre bajo el apoyo didáctico, material, logístico y humano de su tutor y del grupo de investigación, mediante los cuales pudieron resolver las dudas y problemáticas tecnológicas o didácticas que surgieron. Según avanzaron las sesiones el número de consultas fue disminuyendo, aumentando su autonomía, guiando a los escolares y resolviendo sus dudas. Esto supuso que "ascendieran" de usuarios a creadores y gestores, valorando el aprendizaje y trabajo que es necesario para realizar estas actividades en un entorno real: 
[¿Has sido autosuficiente a la hora de crear actividades tecnológicas durante el Prácticum?]

Estudiante: gracias al cursillo y a vuestra ayuda sí.

Estudiante: creo que he sido autosuficiente pero contar con ayuda hace que las actividades desarrolladas se enriquezcan. [Cuest2]

A partir del curso formativo, los estudiantes universitarios buscaron herramientas no empleadas en la asignatura EFMN que enriquecieran el proceso educativo. La profundidad del aprendizaje fue muy intensa y motivante ya que no se conformaron con los recursos aportados inicialmente, sino que integraron los conocimientos adquiridos para buscar otros que apoyaran sus objetivos y contenidos didácticos. Es decir, los estudiantes partieron de los conocimientos adquiridos en la formación y exploraron respuestas ante las incertidumbres, tal y como expresa Schön (1992) en sus estudios cuando evidencia la búsqueda de nuevas respuestas en el contexto escolar durante el Prácticum. Extraemos que además de producirse una aplicación en el ámbito escolar de la formación tecnológica adquirida, los estudiantes abrieron su abanico de conocimientos y herramientas tecnológicas indagando por cuenta propia. Esto se evidencia en el siguiente extracto: "A raíz del curso de formación he sido capaz de diseñar mis propios materiales para la puesta en práctica de mi intervención." [Doc5]

A pesar de que este curso formativo fue satisfactorio para los estudiantes de Prácticum, indicaron que sería mejorable si tuviera lugar a lo largo de más tiempo para poder profundizar mejor en el uso y creación de actividades con las herramientas tecnológicas, debido a su complejidad inicial.

\subsection{APLICACIÓN DIGITAL EN PRIMARIA}

En este apartado profundizamos en los beneficios y dificultades que supuso la integración de las tecnologías dentro de un aprendizaje ubicuo durante el Prácticum. Como hemos detallado en la segunda sección, en los Centros de Educación Primaria 1 y 3 se realizaron actividades tecnológicas muy concretas que apoyaron los contenidos y objetivos en un determinado momento o sesión, mientras que en el Centro 2 se produjo una integración a lo largo de toda la Unidad Didáctica. Cada estudiante de prácticas evolucionó y respondió de manera distinta a la aplicación e integración de las TIC en el contexto escolar, surgiendo por tanto beneficios y dificultades diversas, tal y como detallamos en la Figura 7.

En este sentido, el primero de los beneficios comunes que hubo en los Centros de Educación Primaria fue una mejora de la competencia digital en los escolares. Aunque el alumnado universitario percibió una mejora competencial, nosotros pudimos valorar con una mayor profundidad su evolución en el Centro Escolar 2, debido al mayor número de intervenciones a lo largo del desarrollo de la Unidad Didáctica, al interés del estudiante de Prácticum por realizar un TFG de corte tecnológico, y también al apoyo de la tutora del centro escolar. Así, los escolares se desenvolvieron progresivamente con las TIC obteniendo una mayor autonomía tecnológica, siempre guiados por el estudiante de prácticas, y mostraron interés en seguir utilizando estas herramientas en la clase de Educación Física. Esta evolución estuvo dificultada por el rol dominante de algunos estudiantes de Educación Primaria que eran líderes en el uso tecnológico y también social, por lo que hubo que cuidar el reparto de los roles y la organización grupal para que los escolares más competentes digitalmente 
colaboraran con el resto y que todos utilizaran los recursos tecnológicos. Esto se evidencia en el siguiente extracto del TFG de uno de los estudiantes de Prácticum: "El alumno posesivo de recursos digitales tiene el control total sobre el dispositivo y limita el aprendizaje del resto de compañeros." [Doc5]

Por otro lado, existió ubicuidad en el aprendizaje, tanto en espacios físicos (en aulas ordinarias y naturales, en los patios y en las calles, etc.) como virtuales. Esto supone una paradoja ya que popularmente se piensa que la tecnología reduce la motricidad, y durante la investigación se evidenció que favoreció la movilidad física entre espacios, tanto físicos como virtuales, apoyado por la portabilidad de los dispositivos móviles. Así, la inclusión de las tecnologías ubicuas en las clases de Educación Primaria provocó una mayor motivación por el aprendizaje e interés por los contenidos y actividades, de manera análoga a los estudios de Pérez-Sanagustín et al. (2011) y Fombona, Goulão, y García (2014), cuyos estudios muestran el relevante atractivo que tienen estos recursos para el alumnado. Esto fue debido a que resultaron más motivantes las actividades que incluyeron TIC y a que no solían utilizarlas en la escuela. A continuación, lo evidenciamos en el siguiente extracto del TFG de uno de los estudiantes: "Éstas [las TIC] han tenido un papel protagonista, desde el momento en que en el primer ciclo no es muy habitual trabajar con tablet, códigos QR... Esto resultó un factor muy motivador en los alumnos." [Doc3]

De manera específica, el estudiante de Prácticum del Centro de Primaria 2 valoró el apoyo de las tecnologías en el campo de la EF ya que este tipo de actividades se adaptaron a otros contenidos de la materia así como a otras disciplinas; ofrecieron estas herramientas un apoyo al proceso evaluativo, como fue la realización de cuestionarios de Google Drive a través de códigos QR; apoyaron la labor docente en la organización de los contenidos y en el desarrollo de los mismos, como por ejemplo la app Wikiloc para elaborar, registrar y mostrar a los escolares la ruta de senderismo a realizar o Eduloc para favorecer la orientación en el espacio; fomentaron el aprendizaje dialógico a través de la interacción y comunicación entre los escolares que tenían mejor competencia digital con los que no la tenían; y finalmente, estas herramientas potenciaron la evaluación del aprendizaje en el momento, de manera inmediata.

Por otro lado, también emergieron problemáticas que se fueron solventando. Tal y como mencionan Tejada y Ruiz (2013), la finalidad es que el alumnado esté en un entorno real educativo, perciba las potencialidades, las dificultades, desarrolle intervenciones, interactúe con los escolares, etc., todo ello en consonancia con el contexto. Esto suscitó la aparición de temores entre el alumnado de Prácticum - además del reto que supone para un estudiante ser novel, realizar actividades en el medio natural y además con tecnología -, como que los escolares no supiera realizar determinadas actividades, o que requiriera de un mayor tiempo de preparación o explicación tecnológica. Otro estudiante de Prácticum (Centro 3) sintió que las actividades tecnológicas podían desvirtuar los aprendizajes motrices, y temor a incluir tecnología además de los contenidos de bici en el aula de EF, a pesar de que reconocía que son un gran apoyo en el aprendizaje, rebajando finalmente el número de actividades tecnológicas en su Unidad Didáctica. Respecto a los estudiantes de Prácticum del Centro 1 , no desarrollaron situaciones reales de enseñanza/aprendizaje ya que apoyaron las clases con varios responsables (compañeros/as de prácticas, investigadores, etc.). Ante esta situación el tutor del Centro Escolar les indicó que organizaran y estructuraran la clase pensando en la situación real de un maestro en el día a día. Este hecho y ante el temor a la excesiva ratio de 
escolares por responsable y dispositivo móvil disponible, provocó que durante la sesión final no se realizaran casi actividades tecnológicas:

En el intermedio el profesor indica al estudiante de Prácticum que reduzca la explicación del inicio y que haga situación real, es decir, que les diga cómo se utiliza el iPad y el escaneo de QR en clase porque de manera normal no se va a encontrar con tanto apoyo docente y de observadores. [Obs2]

Debido a estas primeras experiencias con tecnología ubicua de los escolares, surgieron dificultades respecto al uso de herramientas, que tras la explicación de los estudiantes de Prácticum fueron solventadas. Las problemáticas del Centro Escolar 2 giraron en torno a la adaptación tecnológica a las aplicaciones, la mala comprensión lectora que provocó que algunas actividades tuvieran que rehacerse, la apropiación de las tablets por parte de los líderes tecnológicos, así como algunos problemas de red. En el Centro Escolar 1 los problemas fueron la adaptación tecnológica a las aplicaciones, un excesivo uso de las tablets por algunos escolares, la mala lectura de los QR en superficies curvas y el tipo de letra, la cual fue poco legible para alumnado de $1^{\circ}$ y $2^{\circ}$ de Primaria, ya que están acostumbrados a un trazo continuo que facilite la comprensión de la unidad de la palabra.

Figura 7 - Beneficios y dificultades en los Centros de Educación Primaria

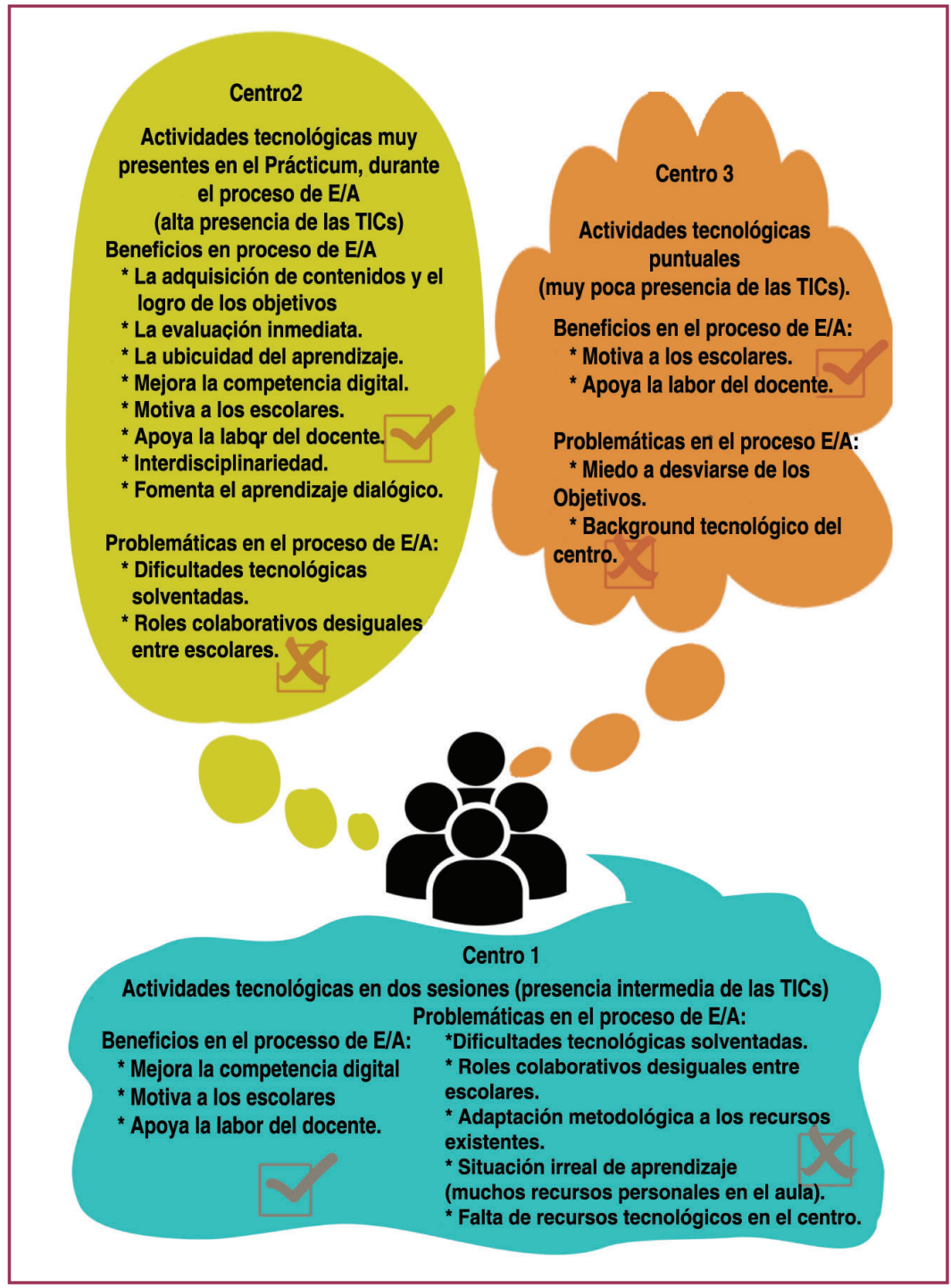

Fuente: elaboración propia 


\subsection{TICS EN LA ESCUELA}

En este apartado analizamos la posibilidad de implementar tecnología en los centros escolares tras la experiencia e investigación realizada, así como los aspectos didácticos a tener en cuenta en el desarrollo del proceso educativo y tecnológico.

En relación con la posible integración de las TIC dentro de los contenidos de EFMN, se evidenció que sí que hubo coexistencia y complementación con la tecnología ubicua, intentando no perder la esencia de la Educación Física en los entornos naturales ni la pérdida de momentos de actividad motriz. En este sentido, los estudiantes de Prácticum regularon la presencia tecnológica en las sesiones teniendo en cuenta la implicación motriz y tecnológica durante las actividades, adoptando para ello las estrategias didácticas adecuadas para cada sesión, con la finalidad de aprovechar los beneficios pedagógicos que aportan las tecnologías. A continuación lo evidenciamos por medio del siguiente extracto del TFG de un estudiante:

[...] las TIC son un recurso integrable en el área de Educación Física y un medio excelente para facilitar su aprendizaje. [...] las nuevas características que se le otorgan a la tecnología como su portabilidad y ubicuidad permiten utilizar este recurso pedagógico de una manera significativa en la Educación Física en el medio natural. [Doc5]

Por otro lado, las evidencias mostraron que a pesar de los temores presentados por los estudiantes, es posible la combinación de tecnología que potencie la ubicuidad dentro de estos contenidos, ya que facilitó el aprendizaje, proporcionó accesibilidad a la información, y apoyó los diversos contenidos, así como otros beneficios mencionados anteriormente, de manera análoga a las investigaciones de Castro-Lemus y Gómez (2016); Muñoz-Cristóbal et al. (2015); y Gallego-Lema et al. (2017) en los cuales dan soporte a contenidos de orientación; y Monguillot et al. (2014) en contenidos de fuerza resistencia. El alumnado de Prácticum también valoró que debería implementarse en la escuela, siempre que fuera oportuno, no solo en contenidos de EFMN sino también en otros del área o incluso en otras asignaturas, pero siempre teniendo en cuenta que es un recurso más a tener presente, que en ningún caso sustituye a otros, y que es de suma importancia integrarlo en la forma y en el momento adecuado:

[¿Crees que en la escuela debe implementarse tecnología dentro de Educación Física en el Medio Natural?]

Si, como complemento de los contenidos, nunca sustituyéndolos.

No, la tecnología es un recurso más que el docente tiene a su alcance, y su uso demuestra una serie de beneficios en el aprendizaje pero es el docente el que tiene que tomar la decisión de integrarlas o no en sus propuestas.

Al igual que pienso que se puede aumentar e incluir las tecnologías en el ámbito de la Educación Física en el medio natural pienso que se debe hacer porque tiene grandes ventajas para todos. [Cuest2]

Para el correcto desarrollo de estas actividades durante el periodo de Prácticum, tuvieron en cuenta diversos aspectos didácticos con la finalidad de que la implementación tecnológica fuera adecuada. En primer lugar fueron previsores con dichas herramientas, la red/cobertura disponible, etc.. Las tablets fueron aportadas por los investigadores, además de algunos iPads que utilizaban habitualmente uno de los Centros Escolares.

De acuerdo con lo comentado anteriormente, se tuvo en cuenta que las actividades con tecnología ubicua fueran colaborativas y que aquellas personas con mayor liderazgo 
cooperaran con los compañeros. En este sentido, se produjo una ayuda informal entre los escolares sobre cómo funcionaban las aplicaciones móviles y los recursos tecnológicos, interacción que Twidale (2010) en sus estudios denomina "Over the Shoulder Learning (OTSL)". También la metodología fue importante para que tecnología y EFMN pudieran caminar de la mano y no desvirtuar la asignatura, apoyando el proceso educativo. Fue relevante no perder de vista el objetivo e ir conjugando las herramientas en el preciso momento. Otro aspecto fue la temporalización de la sesión, sabiendo distribuir bien los tiempos de explicación para no perder mucho rato previo a la actividad, así como calcular bien los tiempos para poder terminarlas. En ciertos momentos en el Centro 2 hubo un excesivo tiempo de parada durante las actividades. El estudiante de Prácticum fue consciente de este detalle y adaptó satisfactoriamente esta situación, tal y como expresa en su TFG:

Las TIC no deben sustituir la actividad motriz. Los recursos digitales son un medio para lograr los aprendizajes del área pero en ningún momento el manejo de estos instrumentos debe reducir la actividad física. Como comprobamos en el análisis, las TIC repercuten en el tiempo destinado a la actividad física, por esta razón debemos intentar disminuir el impacto que producen en el tiempo de actividad. [Doc5]

\section{CONCLUSIONES}

Los resultados del estudio muestran que a partir de la formación base que recibieron los estudiantes universitarios durante la asignatura EFMN y del curso ad hoc tecnológico previo al periodo de prácticas, aplicaron los conocimientos adquiridos a esta etapa (Prácticum) realizando autoaprendizajes para buscar otras herramientas que apoyaran sus contenidos y objetivos didácticos. De esta manera, el alumnado fue evolucionando según transcurrían las sesiones solventando las dudas tecnológicas, sabiendo guiar al alumnado escolar, así como diseñando diversas actividades con TIC. Estas herramientas facilitaron el aprendizaje de los contenidos de EFMN en la escuela, pudiéndose implementar en el aula, teniendo en cuenta que es un recurso más a tener presente y a integrar en el momento adecuado, sin desvirtuar el objeto de aprendizaje. Así, los estudiantes transfirieron la formación recibida a la escuela adaptándola a su pensamiento, al contexto y al currículum, siendo esta integración tecnológica variable entre los tres Centros de Educación Primaria según el background tecnológico del centro, la adaptación de recursos y metodología, los miedos de los estudiantes, el apoyo de los recursos al contenido, y las reflexiones de los estudiantes sobre la coexistencia de la EF con la tecnología para no desvirtuar los aprendizajes motrices. Aparte de estas limitaciones, también emergieron otras a lo largo del estudio, como fueron las de carácter tecnológico: la comprensión de las aplicaciones, la mala lectura de los códigos QR, la batería, etc.; y problemas no tecnológicos, como por ejemplo la dificultad del alumnado de primer ciclo para leer el texto digital debido al tipo de letra.

A lo largo de las sesiones, el aprendizaje ubicuo apoyó los procesos educativos en las escuelas a nivel competencial, en la motivación del alumnado escolar, en la adquisición de contenidos y objetivos, en la evaluación, etc. También apoyó un trabajo de contenidos en distintos lugares y momentos, ayudando a conectar espacios educativos tanto en la creación de contenidos como en su puesta en práctica. Esto supone una paradoja ya que existe el preconcepto de que la tecnología reduce la motricidad cuando en el estudio de caso realizado 
se favoreció la movilidad física en distintos espacios. Por último, incidir en que el diseño llevado a cabo puede ser transferible a otras áreas y adaptable a otros contenidos de Educación Física, favoreciendo la interdisciplinariedad y la intradisciplinariedad.

La investigación desarrollada pone de manifiesto el apoyo que puede brindar la integración de un aprendizaje ubicuo en la formación inicial de profesorado, teniendo presente también las limitaciones que la tecnología otorga en el campo de la educación para (re)pensar la formación de los futuros docentes.

\section{REFERÊNCIAS}

BUNK, Gerhard. La transmisión de las competencias en la formación y perfeccionamiento profesionales de la RFA. Revista Europea de Formación Profesional, n. 1, p. 8-14, 1994.

BURBULES, Nicholas C. Ubiquitous Learning and the Future of Teaching. Encounters on education=Encuentros sobre educación=Recontres sur l'éducation, n. 13, p. 3-14, 2012.

CASTRO-LEMUS, Nuria; GÓMEZ, Iván. Incorporating QR codes in Physical Education in Secondary. Retos: nuevas tendencias en educación física, deporte y recreación, n. 29, p. 114119, 2016.

COPE, Bill; KALANTZIS, Mary. Ubiquitous learning. Urbana: University of Illinois , 2010.

FERNÁNDEZ, Ricardo; HERRERA-VIDAL, Ignacio; NAVARRO, Rubén. Las TIC como recurso en la didáctica de la Educación Física escolar. Propuesta práctica para la Educación Primaria. EmásF: Revista Digital de Educación Física, n. 35, p. 58-69, 2015.

FOMBONA, Javier; GOULÃO, María de F.; GARCIA, Marco A. Melhorar a atratividade da informação através do uso da realidade aumentada. Perspectivas Em Ciência Da Informação, v. 19, n.1, p. 37-50, 2014.

GALLEGO-LEMA, Vanesa et al. Aprendizaje ubicuo: un proceso formativo en Educación Física en el medio natural. Revista Latinoamericana de Tecnología Educativa - RELATEC, v. 15, n. 1, p. 59-73, 2016.

GALLEGO-LEMA, Vanesa et al. La orientación en el medio natural: aprendizaje ubicuo mediante el uso de tecnología. Movimento, v. 23, n. 2, p. 755-770, 2017.

GONZÁLEZ, Gustavo; BARBA, José Juan; RODRíGUEZ, Henar. La importancia del aprendizaje reflexivo en el Prácticum de Magisterio: una revisión de la literatura. Revista de Docencia Universitaria, v. 13, n. 3, 147-170, 2015.

GUTIÉRREZ, Alfonso; PALACIOS, Andrés; TORREGO, Luis. School teacher training and ICT integration in education: anatomy of a mismatch. Revista de educación, n. 353, p. 267-293, 2010.

JORRÍN-ABELLÁN, Iván M.; STAKE, Robert E. Does Ubiquitous Learning Call for Ubiquitous Forms of Formal Evaluation? An Evaluand Oriented Responsive Evaluation Model. Ubiquitous Learning: an International Journal, v. 1, n. 3, 71-82, 2009. 
MARGALEF, Leonor; ÁLVAREZ, Juan Manuel. La formación del profesorado universitario para la innovación en el marco de la integración del espacio europeo de educación superior. Revista de Educación, n. 337, 51-70, 2005.

MINISTERIO DE EDUCACIÓN, CULTURA Y DEPORTE. La integración del sistema universitario español en el espacio europeo de enseñanza superior. Documento-Marco, 2003.

MONGUILLOT, Meritxell et al. Una experiencia colaborativa mediante códigos QR. Revista de Universidad y Sociedad del Conocimiento, v. 11, n. 1, p. 175-191, 2014.

MUÑOZ-CRISTÓBAL, Juan A. Supporting teacher orchestration of across-spaces learning situations. (Tesis doctoral) - Universidad de Valladolid, Valladolid, 2015.

MUÑOZ-CRISTÓBAL, Juan A. et al. Supporting teacher orchestration in ubiquitous learning environments: a study in K-12 Education. Transactions on Learning Technologies, v. 8, n. 1, p. 83-97, 2015.

PÉREZ-SANAGUSTÍN, Mar et al. Discovering the campus together: a mobile and computer-based learning experience. Journal of Network and Computer Applications, v. 35, n. 1, p. 167-188, 2011.

PÉREZ, Francisco; VÍLCHEZ, José E. Perception of teachers trainees about the potential of information technology in education: from expectation to reality. Fuentes: Revista de la Facultad de Ciencias de la Educación, n. 13, p. 155-172, 2013.

PRAT, Queralt; CAMERINO, Oleguer; COIDURAS, Jordi. Introducción de las TIC en educación física. Estudio descriptivo sobre la situación actual. Apunts: Educación Física y Deportes, $n$. 113, p. 37-44, 2013.

QUEROL, Santiago. EF 3.0, una aplicación para educación física. Tándem: Didáctica de la Educación Física, n. 53, p. 33-37, 2016.

RIVERO, Carol et al. Las TIC en la formación universitaria. Revista de Psicología, v. 34, n. 1, p. 185-199, 2016.

SANCHO-GIL, Juana M.; CORREA-GOROSPE, José M. Aprender a enseñar: la constitución de la identidad del profesor en la Educación Infantil y Primaria. Movimento, v. 22, n. 2, p. 471-484, 2015.

SCHÖN, Donald A. La formación de profesionales reflexivos: hacia un nuevo diseño de la enseñanza y el aprendizaje en las profesiones. Barcelona: Paidós, 1992.

STAKE, Robert. Multiple Case Study Analysis. New York: The Guilford Press, 2005.

TEJADA, José; RUIZ, Carmen. Significación del prácticum en la adquisición de competencias profesionales que permiten la transferencia de conocimiento a ámbitos propios de la acción docente. Profesorado. Revista de Currículum y Formación de Profesorado, v. 17, n. 3, p. 91-110, 2013.

TWIDALE, Michael B. From ubiquitous computing to ubiquitous learning. In: B. COPE, Bill; KALANTZIS, Mery (org.), Ubiquitous Learning. Urbana: University of Illinois Press, 2010. p. 72-89.

VÁZQUEZ-CANO, Esteban; SEVILLANO, María Luisa. Dispositivos digitales móviles en educación: el aprendizaje ubicuo. Madrid: Narcea, 2015.

ZABALZA, M. A. El practicum en la formación universitaria: Estado de la cuestión. Revista de Educación, n. 354, p. 21-43, 2011. 


\section{Apoyo:}

Financiación parcial de la Comisión Europea (proyecto 588438-EPP-1-2017-1-EL-EPPKA2-KA), del Ministerio de Economía y Competitividad (proyectos TIN2014-53199-C3-2-R y TIN201785179-C3-2-R); de la Junta de Castilla y León (proyecto VA257P18); y con la ayuda postdoctoral "Especialización de personal investigador doctor" de la Universidad del País Vasco (UPV/EHU).

\section{Agradecimientos:}

Los autores agradecen la colaboración de los estudiantes y centros de Educación Primaria involucrados en esta investigación, así como el apoyo del grupo de investigación GSIC-EMIC, especialmente a Henry Díaz. 